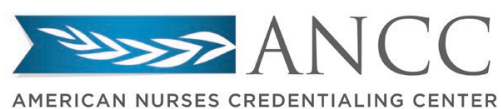

Test Content Outline

Effective Date: January 15, 2020

\author{
Gerontological Nursing \\ Board Certification Examination
}

There are 150 questions on this examination. Of these, 125 are scored questions and 25 are pretest questions that are not scored. Pretest questions are used to determine how well these questions will perform before they are used on the scored portion of the examination. The pretest questions cannot be distinguished from those that will be scored, so it is important for a candidate to answer all questions. A candidate's score, however, is based solely on the 125 scored questions. Performance on pretest questions does not affect a candidate's score.

This Test Content Outline identifies the areas that are included on the examination. The percentage and number of questions in each of the major categories of the scored portion of the examination are also shown. Note: The examples in parentheses are not all-inclusive and do not indicate importance.

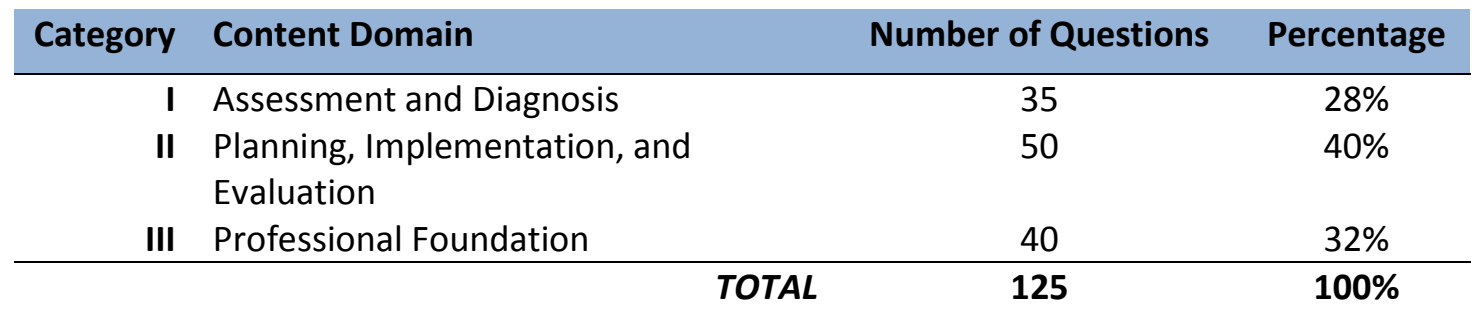




\section{Assessment and Diagnosis}

A. Knowledge

1. Assessment tools and techniques for physical examination and psychosocial assessment (e.g., fall risk, depression screening, pressure injury risk)

2. Pharmacotherapy (e.g., interactions, polypharmacy)

3. Age- and disease-related factors that affect clinical findings

B. Skill

1. Age-related physiological change identification (e.g., sensory, neurological, circulatory)

2. Risk factor identification (e.g., cognitive impairment, non-adherence, abuse and neglect)

\section{Planning, Implementation, and Evaluation}

A. Knowledge

1. Evidence-based interventions and expected outcomes

2. Treatment modality contraindications (e.g., food, drug, herbal supplements)

3. Unique medication needs of older adults (e.g., dosage, route modification, Beers Criteria)

B. Skill

1. Nursing diagnosis and problem list evaluation (e.g., individual preferences, patient-centered care, condition prioritization)

2. Safety intervention implementation

3. Therapeutic environment creation (e.g., individual risk factors, milieu management)

4. Care coordination across the continuum (e.g., case management, transitions, resources, support systems) 


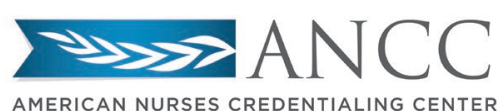

\section{Professional Foundation}

A. Knowledge

1. Theoretical frameworks (e.g., coping, developmental, hierarchy of needs)

2. Disease prevention and health promotion (e.g., screenings, education)

3. Self-management strategies (e.g., home monitoring, telehealth, contingency planning)

4. Principles of adult learning and communication (e.g., preferences, barriers)

5. Diversity and subpopulations of older adults (e.g., grand-families, addictive disorders, veterans, homeless)

6. Legal and ethical considerations (e.g., advance directives, advocacy, research, scope and standards of practice)

7. Regulatory compliance and quality improvement (e.g., Centers for Medicare \& Medicaid Services, The Joint Commission)

Last Updated: November 2, 2018 